\title{
Stannous Fluoride Effects on Enamel: A Systematic Review
}

\author{
Luca Fiorillo $^{1,2, *} \mathbb{C}$, Gabriele Cervino ${ }^{1} \mathbb{C}$, Alan Scott Herford ${ }^{3}$, Luigi Laino ${ }^{2}$ and Marco Cicciù ${ }^{1}$ \\ 1 Department of Biomedical and Dental Sciences, Morphological and Functional Images, \\ University of Messina, Policlinico G. Martino, Via Consolare Valeria, 98100 Messina, Italy; \\ gcervino@unime.it (G.C.); mcicciu@unime.it (M.C.) \\ 2 Multidisciplinary Department of Medical-Surgical and Dental Specialties, University of Campania \\ "Luigi Vanvitelli", 80100 Napoli, Italy; luigi.laino@unicampania.it \\ 3 Department of Maxillofacial Surgery, Loma Linda University, Loma Linda, CA 92354, USA; aherford@llu.edu \\ * Correspondence: lfiorillo@unime.it
}

Received: 19 June 2020; Accepted: 25 August 2020; Published: 31 August 2020

check for updates

\begin{abstract}
In recent years there has been a lot of talk about toothpastes with a particular chemical compound: stannous fluoride $\left(\mathrm{SnF}_{2}\right)$. Its presence is currently still highly controversial, as the latter could have negative health effects. The different companies that produce toothpastes express its dosage in ppm. The purpose of this systematic literature review is to analyze all randomized clinical trials in the literature over the last 10 years and to draw clear results on the function of stannous fluoride, for this purpose the authors performed a Mann-Whitney $U$ Test. Materials: The first analysis of the literature produced a number greater than 800 results, subsequently applying the inclusion and exclusion criteria, and following a manual analysis of the results, 26 manuscripts have been obtained. Results: From the results analyzed in this review, it could be shown that stannous fluoride does not present important contraindications, if not those commonly reported for fluorine. A meta-analysis on enamel loss has been conducted, it shows that $\mathrm{SnF}_{2}$ products provide better results with a $p<0.05$ value. Conclusion: This compound could have significant effects in favor of erosion and recalcification of the enamel, on the biofilm formation, gingival inflammation, and in addition, it could be an important aid in the removal of tooth stains and halitosis.
\end{abstract}

Keywords: dentistry; stannous fluoride; toothpaste; enamel; oral health; oral hygiene

\section{Introduction}

\subsection{Rationale}

Toothpaste is a product aimed at cleaning, maintaining the aesthetics and health of the teeth. Together with the toothbrush it is commonly used to promote oral hygiene. The main functions of this product concern: the removal of food residues from the teeth, the support for the elimination and/or masking of halitosis, the prevention of gum and dental disease, when it also consists of active ingredients such as fluorine or xylitol [1,2]. They represent vehicles through which various active ingredients are applied on the dental surfaces, including antibacterial substances (triclosan, chlorhexidine, cetylpyridinium chloride), bleaching agents (perlite, etc.), desensitizers (amine fluoride), with anti-tartar action (pyrophosphates) or with remineralizing action (stannous fluoride, calcium, phosphates) [3]; remineralizing agents could also be materials that release ions in the mouth (calcium phosphate minerals, bioglass, fluorine salts, etc.) or materials that attach to enamel surface and remineralize it (apatites, amorphous calcium phosphates, etc.). However, it should be noted that they have no action on the removal of the plaque, which occurs exclusively through the mechanical action of 
the brush [4,5]. Traditionally, toothpaste is presented as a cream (also called toothpaste paste), but it is also marketed in the form of a gel, or with mixed compositions. It is generally extracted from a flexible plastic tube. Its use is almost always carried out by placing a portion of this on a toothbrush (both manual and electric), and through the latter it is spread on the dental arches and between the gums. Chemically it is a sol [6,7].

However, toothpastes or powders did not enter general use until the 19th century. The Greeks, and later the Romans, improved the recipes for toothpaste by adding abrasives such as crushed bones and oyster shells [8]. In the ninth century, the Persian musician Ziryab was known to have invented a type of toothpaste, which he popularized throughout Islamic Spain. The precise ingredients of this preparation are currently unknown, but some sources attest that it was "functional and pleasant to taste". It is not known whether these first toothpastes were used alone; it is likely that they were rubbed on the teeth with rags, or used together with early toothbrushes, such as tree twigs. In the 16th century, a US and British toothpaste recipe was found that had burnt bread as an ingredient. Another formula from the same period required dragon blood (a resin), cinnamon and burnt alum. In the twentieth century a paste made of hydrogen peroxide and sodium bicarbonate was recommended for use together with the toothbrush. Pre-packaged toothpastes were marketed in the 19th century, but did not exceed the popularity of tooth powder until the First World War. In 1892, Dr. Washington Sheffield of New London developed toothpaste contained in a collapsible tube. This idea was then copied and applied by numerous manufacturers. Fluoride was added to toothpastes in 1914, and this addition was criticized by the American Dental Association (ADA) in 1937. Fluoride toothpastes developed in 1950 received ADA approval, however [9-11]. Tooth enamel hydroxyapatite is mainly composed of phosphate ions $\left(\mathrm{PO}_{4}{ }^{3-}\right)$ and calcium ions $\left(\mathrm{Ca}^{2+}\right)$. Under normal conditions, there is a stable balance between calcium and phosphate ions in saliva and crystalline hydroxyapatite which makes up $96 \%$ of tooth enamel. When the $\mathrm{pH}$ falls below a critical level (about 5.5 for enamel and 6.2 for dentin), it causes the tooth mineral (hydroxyapatite) to dissolve in a process called demineralization. When the $\mathrm{pH}$ on the tooth surface becomes acidic, the phosphate in oral fluids combines with hydrogen ions $\left(\mathrm{H}^{+}\right)$ to form hydrogen phosphate species (see below). Under these conditions, phosphate is "extracted" from the tooth enamel to restore phosphate levels in saliva and the hydroxyapatite dissolves. When the $\mathrm{pH}$ returns to normal, the calcium and phosphate in saliva can recrystallize into hydroxyapatite, remineralizing the enamel. Fluorine can be administered from several different fluorine sources [12-15]. The three most popular sources of fluoride globally, all of which are accepted by the US FDA as clinically effective, are:

- $\quad$ Stannous fluoride $\left(\mathrm{SnF}_{2}\right)$;

- $\quad$ Sodium fluoride $(\mathrm{NaF})$;

- $\quad$ Sodium monofluorophosphate $\left(\mathrm{Na}_{2} \mathrm{PFO}_{3}\right.$ or SMFP).

The efficacy of fluoride as a caries preventive agent largely depends on its concentration and availability in oral fluids to influence the demineralization/remineralization balance.

Although Stannous Fluoride also has the potential to provide benefits related to the antibacterial properties of the ingredient, this initial formulation provided only a benefit for anticaryosis based on the action of the fluoride. The presence of bioavailable fluoride in the oral fluids (i.e., biofilm and saliva) greatly enhances the crystallization of fluorapatite into tooth structure from calcium and phosphate ions present in saliva. Stannous fluoride adheres to the surface of tooth enamel and forms a protective layer that is able to shield enamel from the effects of erosive acids (Figure 1). Sodium fluoride is a fluoride salt commonly used in dentifrices and oral rinses. Sodium fluoride delivers a highly reactive fluoride ion; therefore, formulating it with a compatible abrasive is critically important for achieving the anticaries benefit. Unlike sodium fluoride, Sodium Monophosphate is not an ionic fluoride salt, but rather a covalently bound compound that requires enzymatic activation by a salivary enzyme (alkaline phosphatase) to release bioavailable fluoride. Because of this lower reactivity, SMFP is compatible with more abrasives than other fluoride sources [16]. 


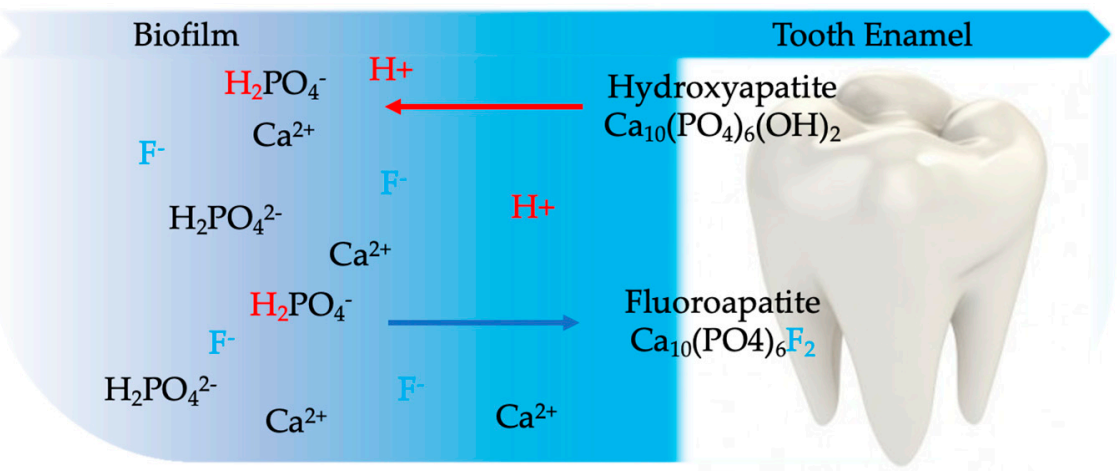

Figure 1. When the $\mathrm{pH}$ drops below 5.5, the biofilm fluid becomes undersaturated with phosphate ion and enamel dissolves to restore balance. When fluoride $\left(\mathrm{F}^{-}\right)$is present, fluorapatite is incorporated into demineralized enamel and subsequent demineralization is inhibited.

Fluoride in various forms is the most popular active ingredient for the prevention of tooth decay in toothpastes [17-19]. Although present in small quantities in plants, animals, and in some sources of natural water, as well as having effects on the formation of tooth and bone enamel, it is not considered an essential element of the diet and there are no known signs of pathological deficit in its absence. Sodium fluoride $(\mathrm{NaF})$ is the most common form; some brands use sodium monofluorophosphate $\left(\mathrm{Na}_{2} \mathrm{PFO}_{3}\right)$ or amine fluoride $297\left(\mathrm{C}_{27} \mathrm{H}_{60} \mathrm{~F}_{2} \mathrm{~N}_{2} \mathrm{O}_{3}\right)$. The first toothpaste containing biomimetic synthetic hydroxyapatite appeared in Europe as a valid alternative to fluorine for the remineralization and repair of tooth enamel [20-23]. The function of biomimetic hydroxyapatite is to protect the teeth by creating a new layer around the tooth, hardening the existing enamel which chemically changes to fluorapatite $\left(\mathrm{Ca}_{5}\left(\mathrm{PO}_{4}\right)_{3} \mathrm{~F}\right)$. Biomimetic nano hydroxyapathite has been extensively tested. After nano hydroxyapathite treatment the enamel has been reported to be more mineralized [24] and harder [25] than demineralized enamel. In recent years there has been a lot of talk about a compound, stannous fluoride, its presence in toothpastes is still much debated and the effects are not fully clarified. Stannous fluoride $\left(\mathrm{SnF}_{2}\right)$ is both bacteriostatic and bactericidal and can reduce bacterial growth and control biofilm. There are several active ingredients that are used in remineralizing products: fluorine compounds, calcium phosphates, hydroxyapatite, amorphous forms of calcium phosphate are the most relevant. Fluoride is a mineral that could strengthen teeth and protect them from cavities. It is normally present in drinking water and in some foods (e.g., fish, peanuts and tea), as well as in products for oral hygiene. Useful in the prevention of dental caries, they are local application with the use of fluoride-based toothpastes and mouthwashes, and fluoroprophylaxis treatments during the hygiene sessions in the office. The intake of fluoride, in the form of tablets, can be indicated in the growth phase of the teeth (up to about the age of 12), especially when the drinking water taken daily does not already have an optimal amount of fluoride (from 0,5 to $1.0 \mathrm{mgF} / \mathrm{L}$ ) [26]. However, not all variants used by the industry have the same ability to interact with dental tissues and some are definitely not very effective if not as desensitizers. Various formulas have been presented by toothpaste manufacturers to avoid oxidation, including recently one in which the incorporation of zinc phosphate has been proposed [27-29].

\subsection{Objectives}

The objectives of this review are to highlight all the clinical features concerning stannous fluoride reported in the literature and eventually its chemical interactions.

- In dental patients, what is the effect of stannous fluoride compositions on oral health compared to other dental healthcare products?

And as secondary outcome: 
- On enamel and other hard tooth tissue, what is the effect of stannous fluoride composition on their structure compared to other dental healthcare products?

\section{Materials and Methods}

\subsection{Protocol and Registration}

Well-defined protocols were strictly followed for the preparation of this review. First, a search was conducted in the systematic review databases to highlight similar studies or not. Subsequently the systematic review has been registered on PROSPERO (international database of prospectively registered systematic reviews in health and social care, welfare, public health, education, crime, justice, and international development, where there is a health-related outcome). PROSPERO is produced by CRD and funded by the National Institute for Health Research (NIHR). The number and date of registration (under review) on PROSPERO are as follows: number 176261 on 24/03/2020.

The systematic review was conducted in accordance with the PRISMA (Preferred Reporting Items for Systematic Reviews and Meta-Analyses) statement, all the guidelines were followed (Checklist/Flow diagram), the division into chapters and paragraphs was respected. The analysis of the risk of bias and the setting up of the research, including the drafting of the objective questions of the systematic review, respected the PRISMA criteria, and in particular, in the latter case the PICO (Population/Intervention/Comparison/Outcome) guidelines.

\subsection{Eligibility Criteria}

The full text of all studies of possible relevance was obtained for assessment against the following inclusion criteria:

- Study about stannous fluoride dentifrice/toothpaste/mouth rinse.

- Study of patient side effects of stannous fluoride.

- Study about stannous fluoride chemo-physical interaction.

- Clinical studies on stannous fluoride use and control groups.

- Articles published in the last 10 years.

The applied exclusion criteria for studies were as follows:

- Studies involving subjects with other specific diseases, immunological disorders, oncological patients, osteoporosis, and genetic diseases.

- Not enough information regarding the selected topic.

- No access to the title and abstract in English language or letters, commentary, PhD thesis and editorials.

- $\quad$ Not Randomized Controlled Trial (RCT) studies.

\subsection{Information Sources}

The search strategy has been conducted on different electronic databases. A search on Ovid MEDLINE, PubMed and EMBASE for relevant studies published was carried out. A hand search of the reference lists in the articles retrieved was carried out to highlight any additional publications and to improve the sensitivity.

\subsection{Search}

Search has been conducted using the following keyword "stannous fluoride". The choice of keywords was intended to collect as much relevant data as possible without relying on electronic means alone to refine the search results. The choice of keywords was made in accordance with the MeSH words (Medical Subject Headings). 


\subsection{Study Selection}

Two independent reviewers (L.F. and G.C.) of the University of Messina singularly analyzed the results in order to select inclusion and exclusion criteria. They compared decisions and resolved differences through help of a third expert reviewer (M.C.) For the stage of reviewing of full-text articles, a complete independent dual revision was performed. The results have been compared at the end of the research with a fourth external senior reviewer (A.S.H.). A possible disagreement regarding the inclusion of the studies was discussed among the authors.

\subsection{Data Collection Process}

The first phase of the research consisted of the selection of titles, which allowed us to make a first screening of the manuscript eliminating those not concerning our research. Finally, the full text of all studies was obtained and according to the expected inclusion/exclusion criteria, articles were selected and included in the present review.

\subsection{Data Items}

After the first literature analysis, all article titles were screened to exclude irrelevant publications, case reports, and the non-English language publications. Then, researches were not selected based on data obtained from screening the abstracts. The final stage of screening involved reading the full texts to confirm each study's eligibility, based on the inclusion and exclusion criteria.

\subsection{Risk of Bias in Individual Studies}

This type of review analyses all the studies in the literature in the last ten years presenting a review of recent data about stannous fluoride clinical effects. Regardless of the results of the studies taken into consideration, the evaluation was carried out on the field of action of the analyses carried out by the studies. Risk of bias analysis has been conducted according to PRISMA guidelines [30-32].

\subsection{Summary Measures}

Data were collected from results and arranged in in tables with the following fields (Tables 1-3):

Table 1:

- Authors and Year-Authors and year of publication

- Sample size-Information about sample size and sample type

- Groups-Information about number of groups and type of group (each group is separate by "vs.")

- Time and/or Follow up-Information about timing and follow up of the study

- Main results-Main outcomes and results of the analyzed study

- Statistic results-Statistical results (if performed)

Table 2:

- Main outcome-Classifications of the outcomes obtained by search

- N. of results-Number of obtained results in that outcome set

Table 3:

- Authors and Year-Authors and year of publication

- Sample-Information about sample size and sample type

- Measured at-Obtaining date of the median value

- Mean value-Mean value of groups $\left(\mathrm{SnF}_{2}\right.$ group first vs. other) 
Table 1. Results of individual studies table. This table shows results according to paragraph summary measures.

\begin{tabular}{|c|c|c|c|c|c|}
\hline Authors and Year & Sample Size & Groups & $\begin{array}{l}\text { Time and/or } \\
\text { Follow up }\end{array}$ & Main Results & Statistic Results \\
\hline West et al. [33] 2019 & 36 & $\begin{array}{c}2 \text { Groups: } 0.454 \% \text { stannous fluoride dentifrice vs. } \\
\text { market dentifrice } \mathrm{NaF} / \text { triclosan }(0.24 \% \text { sodium } \\
\text { fluoride and } 0.3 \% \text { triclosan })\end{array}$ & 10 days trial & $\begin{array}{l}\text { Stannous fluoride dentifrice demonstrated } 93.5 \% \\
\text { less enamel loss than control }\end{array}$ & $p<0.001$ \\
\hline $\begin{array}{l}\text { Seriwatanachai et al. [34] } \\
\quad 2019\end{array}$ & 135 & $\begin{array}{l}3 \text { Groups: Stabilized } \mathrm{SnF}_{2} \text { dentifrice vs. } \mathrm{SnF}_{2} \text { with } \\
\text { zinc lactate dentifrice vs. a fluoride dentifrice }\end{array}$ & 6 months & $\begin{array}{c}\text { Both } \mathrm{SnF}_{2} \text { dentifrice showed a statistically } \\
\text { significant reduction of gingival inflammation and } \\
\text { plaque. With no statistical differences between } \\
\text { themselves }\end{array}$ & $p<0.001$ \\
\hline Luo et al. [35] 2019 & 150 & $\begin{array}{l}3 \text { Groups: (48) Potassium nitrate vs. (45) stannous } \\
\text { fluoride vs. (46) placebo }\end{array}$ & 30 days & $\begin{array}{l}\text { Authors demonstrated how Potassium nitrate } \\
\text { toothpaste could reduce sensitivity after an in-office } \\
\text { bleaching treatment, with no differences between } \\
\text { stannous fluoride and placebo. }\end{array}$ & $p<0.05$ \\
\hline Li et al. [36] 2019 & $\begin{array}{l}18 \text { bovine } \\
\text { enamel sample }\end{array}$ & $\begin{array}{l}3 \text { Groups: } 0.454 \% \mathrm{SnF}_{2} \text { and } 1 \% \text { zinc phosphate vs. } \\
\text { Crest Pro-Health Whitening Power vs. non-abrasive } \\
\qquad \mathrm{SnF}_{2} \text { gel }\end{array}$ & 6 weeks & $\begin{array}{l}\text { In this in vitro study } \mathrm{SnF}_{2} \text { and } 1 \% \text { zinc paste } \\
\text { performed better results than competitor and } \\
\text { non-abrasive gel. It showed a better tooth stain } \\
\text { reduction with no adverse effect. }\end{array}$ & $p<0.01$ at 3 weeks \\
\hline Ionta et al. [37] 2019 & $\begin{array}{l}256 \text { bovine } \\
\text { enamel sample }\end{array}$ & $\begin{array}{l}4 \text { Groups: calcium silicate, sodium phosphate, and } \\
1450 \mathrm{ppm} \text { sodium monofluorophosphate vs. } \\
\text { dentifrice with } 3500 \mathrm{ppm} \text { stannous chloride, } \\
700 \mathrm{ppm} \text { amine fluoride, and } 700 \mathrm{ppm} \text { sodium } \\
\text { fluoride vs. conventional dentifrice, with } 1450 \mathrm{ppm} \\
\text { sodium monofluorophosphate vs. control } \\
\text { (deionized water) }\end{array}$ & 20 days & $\begin{array}{l}\text { The group } 1 \text { promoted less enamel loss than water } \\
\text { (group } 4 \text { ) but it did not differ from group } 2 \text { or } 3 \text {. But } \\
\text { group } 1 \text { dentifrice promoted a higher wear after } \\
\text { erosion than other groups. }\end{array}$ & $p<0.05$ \\
\hline Hu et al. [38] 2019 & 100 & 2 Groups: $\mathrm{SnF}_{2}$ dentifrice vs. fluoride dentifrice & 6 months & $\begin{array}{l}\text { Both groups had a significant reduction in gingival } \\
\text { inflammation and a plaque control improvement. } \\
\mathrm{SnF}_{2} \text { dentifrice showed a reduction of all indexed } \\
\text { compared to control dentifrice }\end{array}$ & $p<0.001$ \\
\hline $\begin{array}{l}\text { Haraszthy et al. [39] } \\
2019\end{array}$ & 129 & $\begin{array}{l}2 \text { Groups: Stannous fluoride toothpaste vs. sodium } \\
\text { monofluorophosphate toothpaste }\end{array}$ & 8 weeks & $\begin{array}{c}\text { Stannous fluoride group showed a greater reduction } \\
\text { of bacteria. From } 14 \% \text { at time zero to } 27 \% \text { at } \\
4 \text { weeks, and } 41 \% \text { at } 8 \text {-week time. }\end{array}$ & $p<0.05$ \\
\hline $\begin{array}{l}\text { Hagenfield et al. [40] } \\
\qquad 2019\end{array}$ & 41 & $\begin{array}{l}2 \text { Groups: anti-adhesive zinc-substituted carbonated } \\
\text { hydroxyapatite }(\mathrm{HA}) \text { vs. with antimicrobial and } \\
\text { anti-adhesive amine fluoride/stannous fluoride } \\
\left(\mathrm{AmF} / \mathrm{SnF}_{2}\right)\end{array}$ & 12 weeks & $\begin{array}{c}\text { There were no differences between groups in } \\
\text { microbiome changes. }\end{array}$ & $p>0.05$ \\
\hline
\end{tabular}


Table 1. Cont.

\begin{tabular}{|c|c|c|c|c|c|}
\hline Authors and Year & Sample Size & Groups & $\begin{array}{l}\text { Time and/or } \\
\text { Follow up }\end{array}$ & Main Results & Statistic Results \\
\hline $\begin{array}{l}\text { Creeth et al. [41] } \\
\quad 2019\end{array}$ & 656 & $\begin{array}{l}2 \text { Groups: (329) experimental anhydrous } 0.454 \% \\
\left.\text { SnF }_{2} / \text { polyphosphate toothpaste vs. ( } 327\right) \text { toothpaste } \\
\text { containing } 0.76 \% \text { sodium monofluorophosphate }\end{array}$ & 3 days & $\begin{array}{c}\text { Experimental toothpaste reduced dentine } \\
\text { hypersensitivity (DH) after } 3 \text { days treatment better } \\
\text { than test group. }\end{array}$ & $p<0.0001$ \\
\hline Zero et al. [42] 2018 & 168 & $\begin{array}{c}4 \text { Groups: sodium fluoride }(\mathrm{NaF}) / \mathrm{Carb} / \text { silica, } \\
\text { NaF/silica, NaF }+ \\
\text { monofluorophosphate }(\mathrm{MFP}) / \text { chalk vs. } \\
\mathrm{NaF} / \mathrm{Carb} / \mathrm{silica}, \mathrm{NaF}+\mathrm{MFP} / \mathrm{dical} \text {, amine fluoride } \\
(\mathrm{AmF}) / \text { silica vs. } \mathrm{NaF} / \mathrm{Carb} / \text { silica, NaF }+ \text { stannous } \\
\text { fluoride }\left(\mathrm{SnF}_{2}\right) / \text { silica/hexametaphosphate }(\mathrm{HMP}) \text { vs. } \\
\text { Placebo }(0 \mathrm{ppm} \mathrm{F}) \text { and/or dose-response controls } \\
(675 \mathrm{ppm} \mathrm{F} \text { as NaF }[675 \mathrm{~F}-\mathrm{NaF}]) \pm \mathrm{Carb}\end{array}$ & 14 days & 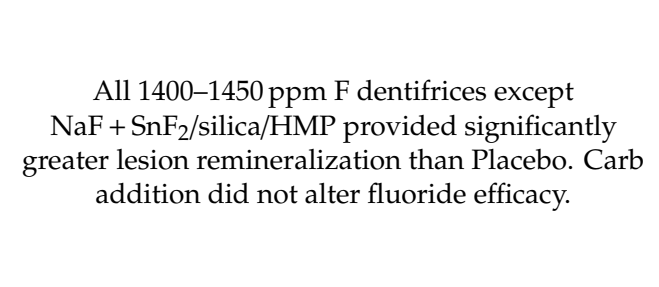 & $p<0.0001$ \\
\hline West et al. [43] 2018 & 21 samples & $\begin{array}{l}3 \text { Groups: toothpaste containing } 0.454 \% \text { stannous } \\
\text { fluoride vs. Control fluoride toothpaste containing } \\
0.76 \% \text { sodium monofluorophosphate vs. mineral } \\
\text { water }\end{array}$ & 10 days & $\begin{array}{l}\text { After } 4 \text { days of treatment the degree of tubule } \\
\text { occlusion increased in the dentine samples in the } \\
\text { groups } 1 \text { and } 2 \text { than in water. }\end{array}$ & $p<0.01$ \\
\hline Frese et al. [44] 2018 & 54 & 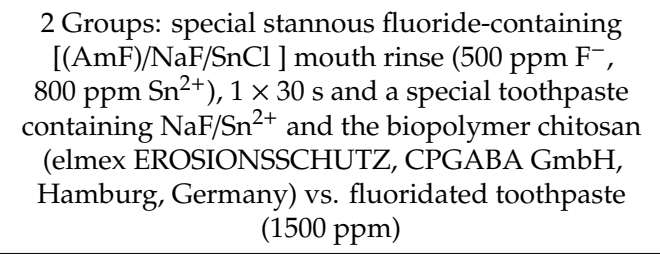 & 4 years & $\begin{array}{l}\text { Two groups showed similar caries prevalence. There } \\
\text { was a decrease of caries superficialis and media. }\end{array}$ & 1 \\
\hline West et al. [45] 2017 & $\begin{array}{l}33 \text { human } \\
\text { enamel sample }\end{array}$ & $\begin{array}{l}2 \text { Groups: } 0.454 \% \mathrm{SnF}_{2} / 0.077 \% \mathrm{NaF} \text { vs. } 0.32 \% \\
\text { NaF/0.3\% triclosan. }\end{array}$ & 15 days & $\begin{array}{l}\mathrm{SnF}_{2} \text { group provided a reduction of enamel loss at } \\
\text { day } 10 \text { and again at day } 15 .\end{array}$ & $p<0.0001$ \\
\hline West et al. [46] 2017 & 33 & $\begin{array}{l}2 \text { Groups: } \mathrm{SnF}_{2}+0.77 \% \text { sodium fluoride }(\mathrm{NaF}) \text { vs. } \\
\text { sodium monofluorophosphate/arginine dentifrice }\end{array}$ & 10 day & $\begin{array}{l}\text { Group } 1 \text { provided better enamel protection against } \\
\text { erosive acid challenge than group } 2\end{array}$ & $p<0.0001$ \\
\hline $\begin{array}{l}\text { Marchetti et al. [47] } \\
\qquad 2017\end{array}$ & 20 & $\begin{array}{l}3 \text { Groups: Alcohol free essential oil mouthwash vs. } \\
\text { Amine fluoride/stannous fluoride with zinc lactate } \\
\text { mouthwash vs. chlorhexidine (CHX) mouthwash }\end{array}$ & 3 days & $\begin{array}{l}\text { Group } 1 \text { showed better results on plaque regrowth } \\
\text { compared to alcohol-free essential oil mouthwash. } \\
\text { But there was a less impact if compared to CHX. }\end{array}$ & $p<0.001$ \\
\hline
\end{tabular}


Table 1. Cont.

\begin{tabular}{|c|c|c|c|c|c|}
\hline Authors and Year & Sample Size & Groups & $\begin{array}{l}\text { Time and/or } \\
\text { Follow up }\end{array}$ & Main Results & Statistic Results \\
\hline $\begin{array}{l}\text { Geidel et al. [48] } \\
\quad 2017\end{array}$ & 76 & $\begin{array}{l}3 \text { Groups: Herbal toothpaste vs. triclosan/copolymer } \\
\text { toothpaste vs. amine/stannous fluoride toothpaste }\end{array}$ & 24 weeks & $\begin{array}{c}\text { Approximal plaque index (API) and Oral hygiene } \\
\text { index (OHI) changed in all groups with } \\
\text { a significantly lower API e OHI in group } 1 \text {. Sulcus } \\
\text { bleeding index (SBI) was improved in all groups } \\
\text { after } 12 \text { weeks. Bleeding on Probing (BoP) was } \\
\text { unchanged. }\end{array}$ & $p=0.001$ \\
\hline $\begin{array}{l}\text { Lorenz et al. [49] } \\
\quad 2015\end{array}$ & 28 & $\begin{array}{l}5 \text { Groups: amine fluoride/stannous fluoride } \\
\left(\mathrm{AmF} / \mathrm{SnF}_{2}\right), 250 \mathrm{ppm} \mathrm{F}^{-} ; \text {low concentration of } \\
\text { film-forming agents; low concentration of } \\
\text { humectants vs. amine fluoride/stannous fluoride, } \\
250 \mathrm{ppm} \mathrm{F}^{-} ; \text {low concentration of film-forming } \\
\text { agents, high concentration of humectants vs. amine } \\
\text { fluoride/stannous fluoride, } 250 \mathrm{ppm} \mathrm{F}^{-} \text {; high } \\
\text { concentration of film-forming agents; high } \\
\text { concentration of humectants vs. Phenolic/essential } \\
\text { oil mouth rinse vs. Volvic Still Water, Danone Waters }\end{array}$ & 10 days & $\begin{array}{l}\text { All mouth rinses led to tooth and tongue staining, } \\
\text { statistically significant differences existed between } \\
\text { groups 1, 3, } 4 \text { and } 5 \text { on tooth staining }\end{array}$ & 1 \\
\hline Hove et al. [50] 2014 & $\begin{array}{l}64 \text { human teeth } \\
\text { sample }\end{array}$ & $\begin{array}{l}4 \text { Groups: Fluoride-free toothpaste vs. toothpaste } \\
0.4 \% \mathrm{SnF}_{2} \text { vs. toothpaste } 0.454 \% \mathrm{SnF}_{2} \text { vs. fluoride } \\
\text { free toothpaste and a } 0.4 \% \mathrm{SnF}_{2} \text { solution } \\
(1000 \mathrm{ppm} \mathrm{F})\end{array}$ & 9 days & $\begin{array}{c}\text { The } \mathrm{SnF}_{2} \text { groups showed significantly lower enamel } \\
\text { wear than the group } 1\end{array}$ & $p<0.05$ \\
\hline $\begin{array}{l}\text { Bellamy et al. [51] } \\
\quad 2014\end{array}$ & 12 & $\begin{array}{l}3 \text { Groups: sodium fluoride dentifrice vs. Stannous } \\
\text { fluoride dentifrice vs. water }\end{array}$ & 15 days & $\begin{array}{l}\text { Enamel loss was significantly lower for treatment in } \\
\text { group } 2 \text { versus } 1 \text { or } 3\end{array}$ & $p<0.005$ \\
\hline $\begin{array}{l}\text { Bellamy et al. [52] } \\
2014\end{array}$ & 27 & $\begin{array}{c}2 \text { Groups: } \mathrm{SnF}_{2} / \text { sodium fluoride }(\mathrm{NaF}) \text { dentifrice vs. } \\
\text { anticavity dentifrice }\end{array}$ & 17 days & $\begin{array}{l}\text { Group } 1 \text { showed better results on } 17 \text { days usage } \\
\text { period, it demonstrated a statistically significant } \\
\text { a lower mean plaque area at each timepoint. }\end{array}$ & $p<0.0001$ \\
\hline $\begin{array}{l}\text { Stenhagen et al. [53] } \\
2013\end{array}$ & $\begin{array}{l}16 \text { molars } \\
\text { sample }\end{array}$ & 4 Groups: $\mathrm{NaF}_{\text {vs. }} \mathrm{SnF}_{2}$ vs. $\mathrm{TiF}_{4}$ vs. control & 9 days & $\begin{array}{l}\text { The mean surface loss in the } \mathrm{NaF}, \mathrm{SnF}_{2} \text { and } \mathrm{TiF}_{4} \\
\text { groups was significantly lower than in the } \\
\text { control group }\end{array}$ & $p<0.05$ \\
\hline $\begin{array}{l}\text { Jentsch et al. [54] } \\
\quad 2013\end{array}$ & 24 & $\begin{array}{l}3 \text { Groups: Essential oil mouth rinse vs. } \\
\text { amine/stannous fluoride mouth rinse vs. } \\
\text { chlorhexidine digluconate } 0.12 \% \text { mouth rinse }\end{array}$ & $96 \mathrm{~h}$ & $\begin{array}{l}\text { The counts of cocci and bacilli and plaque thickness } \\
\text { are statistically different only in chlorhexidine } \\
\text { digluconate } 0.12 \% \text { group, with positive results }\end{array}$ & $p \leq 0.05$ \\
\hline
\end{tabular}


Table 1. Cont.

\begin{tabular}{|c|c|c|c|c|c|}
\hline Authors and Year & Sample Size & Groups & $\begin{array}{l}\text { Time and/or } \\
\text { Follow up }\end{array}$ & Main Results & Statistic Results \\
\hline West et al. [55] 2012 & 20 & 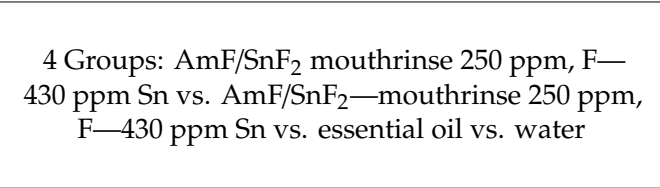 & 4 days & $\begin{array}{l}\text { Rinse } 2 \text { produced less stain than rinse } 1 \text {, but the } \\
\text { difference was not significant. Rinse } 2 \text { produced } \\
\text { significantly more stain than rinse } 3 \text { and } 4 \text {. For } \\
\text { tongue staining, rinse } 2 \text { produced significantly more } \\
\text { staining than } 4 \text { but not } 1 \text { or } 3 \text {. }\end{array}$ & $p<0.05$ \\
\hline Fine et al. [56] 2012 & 35 & $\begin{array}{c}3 \text { Groups: Sodium fluoride/triclosan/copolymer } \\
\text { dentifrice vs. Stannous fluoride/sodium } \\
\text { hexametaphosphate/zinc lactate dentifrice } \\
\left(\mathrm{SnF}_{2} / \mathrm{SHMP}\right) \text { vs. sodium fluoride dentifrice }\end{array}$ & 13 days & $\begin{array}{l}\text { Group } 1 \text { demonstrated significant reduction on } \\
\text { plaque compared to other groups. }\end{array}$ & $p<0.01$ \\
\hline $\begin{array}{l}\text { Huysmans et al. [57] } \\
\qquad 2011\end{array}$ & $\begin{array}{l}20 \text { enamel } \\
\text { samples }\end{array}$ & $\begin{array}{l}3 \text { Groups: } \mathrm{SnF}_{2} \text { toothpaste ( } 1050 \mathrm{ppm} \text { fluoride from } \\
\text { stan- nous fluoride and } 350 \mathrm{ppm} \text { from amine } \\
\text { fluoride) vs. } \mathrm{SnF}_{2} \text { toothpaste (containing } 1100 \mathrm{ppm} \\
\text { fluoride from stannous fluoride and } 350 \mathrm{ppm} \text { from } \\
\text { sodium fluoride) vs. sodium fluoride toothpaste }\end{array}$ & 5 days & $\mathrm{SnF}_{2}$ toothpastes significantly reduced erosive wear. & $p<0.05$ \\
\hline $\begin{array}{l}\text { Wigger-Alberti et al. [58] } \\
\qquad 2010\end{array}$ & 174 & $\begin{array}{c}4 \text { Groups: Amine fluoride/stannous fluoride } 0.2 \% \\
\text { zinc lactate mouthrinse + malodour counteractives } \\
\text { vs. } 0.05 \% \text { CHX, } 0.05 \% \text { cetylpyridinium chloride, } \\
0.14 \% \text { zinc lactate mouthrinse vs. } 0.12 \% \text { CHX } \\
\text { mouthrinse vs. tap water. }\end{array}$ & 21 days & $\begin{array}{l}\text { Group } 1 \text { showed efficacy to teeth discoloration, } \\
\text { a significant reduction of organoleptxic ratings and } \\
\text { volatile sulfur compounds was achieved after single } \\
\text { application and after days } 7 \text { and } 21 \text {. }\end{array}$ & $p<0.001$ \\
\hline
\end{tabular}


Table 2. Risk of bias analysis according to PRISMA, as specified in paragraph risk of bias ("+": low risk of bias; “-“: high risk of bias; "?": unclear risk of bias).

\begin{tabular}{|c|c|c|c|c|c|c|}
\hline Author & $\begin{array}{c}\text { Random Sequence } \\
\text { Generation } \\
\text { (Selection Bias) }\end{array}$ & $\begin{array}{c}\text { Allocation } \\
\text { Concealment } \\
\text { (Selection Bias) }\end{array}$ & $\begin{array}{l}\text { Blinding of Participants } \\
\text { and Personnel } \\
\text { (Performance Bias) }\end{array}$ & $\begin{array}{c}\text { Blinding of Outcome } \\
\text { Assessment } \\
\text { (Detection Bias) }\end{array}$ & $\begin{array}{c}\text { Incomplete } \\
\text { Outcome Data } \\
\text { (Attrition Bias) }\end{array}$ & $\begin{array}{c}\text { Selective } \\
\text { Reporting } \\
\text { (Reporting Bias) }\end{array}$ \\
\hline West et al. [33] 2019 & + & + & + & + & + & + \\
\hline Seriwatanachai et al. [34] 2019 & + & + & + & + & + & + \\
\hline Luo et al. [35] 2019 & + & + & - & - & + & + \\
\hline Li et al. [36] 2019 & + & + & + & + & + & + \\
\hline Ionta et al. [37] 2019 & + & - & + & - & + & + \\
\hline Hu et al. [38] 2019 & + & + & + & - & + & + \\
\hline Haraszthy et al. [39] 2019 & + & + & - & - & + & + \\
\hline Hagenfield et al. [40] 2019 & + & + & + & + & + & + \\
\hline Creeth et al. [41] 2019 & - & - & + & + & + & + \\
\hline Zero et al. [42] 2018 & + & + & - & - & + & + \\
\hline West et al. [43] 2018 & + & + & + & - & + & + \\
\hline Frese et al. [44] 2018 & + & + & - & - & + & + \\
\hline West et al. [45] 2017 & + & + & + & + & + & + \\
\hline West et al. [46] 2017 & + & + & + & + & + & + \\
\hline Marchetti et al. [47] 2017 & + & - & + & + & + & + \\
\hline Geidel et al. [48] 2017 & - & + & - & - & + & + \\
\hline Lorenz et al. [49] 2015 & + & - & + & - & + & + \\
\hline Hove et al. [50] 2014 & - & - & - & - & + & + \\
\hline Bellamy et al. [51] 2014 & + & - & - & - & + & + \\
\hline Bellamy et al. [52] 2014 & + & - & + & + & + & + \\
\hline Stenhagen et al. [53] 2013 & - & - & - & - & + & + \\
\hline Jentsch et al. [54] 2013 & - & - & - & - & + & + \\
\hline West et al. [55] 2012 & + & - & + & - & + & + \\
\hline Fine et al. [56] 2012 & + & + & + & + & + & + \\
\hline Huysmans et al. [57] 2011 & + & + & - & - & - & + \\
\hline Wigger-Alberti et al. [58] 2010 & + & - & + & + & + & + \\
\hline
\end{tabular}


Table 3. Main outcome table as specified in summary measures paragraph.

\begin{tabular}{cc}
\hline Main Outcome & No. of Results \\
\hline Enamel loss reduction $[17,21,29,30,33,34,36,40]$ & 9 \\
\hline $\begin{array}{c}\text { Bacteria and others microorganisms } \\
\text { reduction }[23,24,31,32,35,37,39]\end{array}$ & 7 \\
\hline tooth stain reduction $[20,38,49]$ & 4 \\
\hline Gingival inflammation reduction [18,22,32] & 2 \\
\hline Dentinal hypersensitivity reduction [19,25] & 2 \\
\hline Carious lesion remineralization [26,28] & 2 \\
\hline Dentin tubule occlusion [27] & 1 \\
\hline organoleptic ratings and volatile sulfur compounds [41] & 1 \\
\hline
\end{tabular}

\subsection{Risk of Bias Across Studies}

A risk of bias analysis has been conducted and it is shown in Table 2.

\subsection{Additional Analyses}

Having two random, independent samples authors used a Mann-Whitney $U$ Test ( 0.05 significance level) with the null hypothesis that medians of the two samples are identical. Statistical data is shown in Table 4.

Table 4. Obtained data and meta-analysis on enamel wear loss.

\begin{tabular}{|c|c|c|c|}
\hline Author and Year & Sample & Measured at & Mean Value \\
\hline West et al. 2019 [33] & $\begin{array}{l}36 \text { Intraoral appliances with } \\
\text { human enamel sample }\end{array}$ & Day 10 & $0.097 \mu \mathrm{m}$ vs. $1.495 \mu \mathrm{m}$ \\
\hline Ionta et al. 2019 [37] & 256 Bovine enamel blocks & Day 5 & $4.8 \pm 2.5 \mu \mathrm{m}$ vs. $4.8 \pm 1.4 \mu \mathrm{m}$ \\
\hline West et al. 2017 [43] & $\begin{array}{l}33 \text { Intraoral appliances with } \\
\text { human enamel sample }\end{array}$ & Day 15 & $1.60 \mu \mathrm{m}$ vs. $5.03 \mu \mathrm{m}$ \\
\hline West et al. 2017 [44] & $\begin{array}{l}33 \text { Human enamel } \\
\text { specimens }\end{array}$ & Day 15 & $5.75 \mu \mathrm{m}$ vs. $23.75 \mu \mathrm{m}$ \\
\hline Hove et al. 2014 [50] & $\begin{array}{l}16 \text { Intraoral appliances with } \\
\text { human molar }\end{array}$ & Day 9 & $\begin{array}{c}14.5 \pm 9.3 \mu \mathrm{m} \text { and } \\
33.3 \pm 7.4 \mu \mathrm{m} \text { and } 0.4 \pm 1.3 \mu \mathrm{m} \\
\text { vs. } 29.2 \pm 10.5 \mu \mathrm{m}\end{array}$ \\
\hline Bellamy et al. 2014 [51] & $\begin{array}{l}12 \text { intraoral appliances with } \\
\text { human enamel sample }\end{array}$ & Day 15 & $2.03 \mu \mathrm{m}$ vs. $15.53 \mu \mathrm{m}$ \\
\hline Stenhagen et al. 2013 [53] & $\begin{array}{l}16 \text { intraoral appliances with } \\
\text { molar sample }\end{array}$ & Day 9 & $\begin{array}{c}1.8 \pm 1.9 \mu \mathrm{m} \text { vs. } 26.3 \pm 4.7 \mu \mathrm{m} \\
\text { vs. } 3.1 \pm 4.8\end{array}$ \\
\hline Huysmans et al. 2011 [57] & $\begin{array}{l}20 \text { intraoral appliances with } \\
\text { human enamel sample }\end{array}$ & Day 5 & data not clear or incomplete \\
\hline
\end{tabular}

\section{Results}

\subsection{Study Selection}

From the first research, a total of 833 results were obtained from the scientific databases. Subsequently these results, subjected to screening and application of the inclusion and exclusion criteria, were reduced as follows. Initially only the Randomized Controlled Trial items were selected, obtaining a total of 185 results. Subsequently, only the articles published in the last 10 years were considered, for a total of 76 results remaining. In this review, only the results of the past decade on the effects of stannous fluoride were considered. This aspect, carefully evaluated by the authors, aims to 
be able to compare in vitro or in vivo study techniques similar to each other, and above all chemical formulations as similar as possible. Therefore, only the accessible and available data articles (34) and subsequently the related ones and with sufficient information to conduct a review were considered (26) (Figure 2).

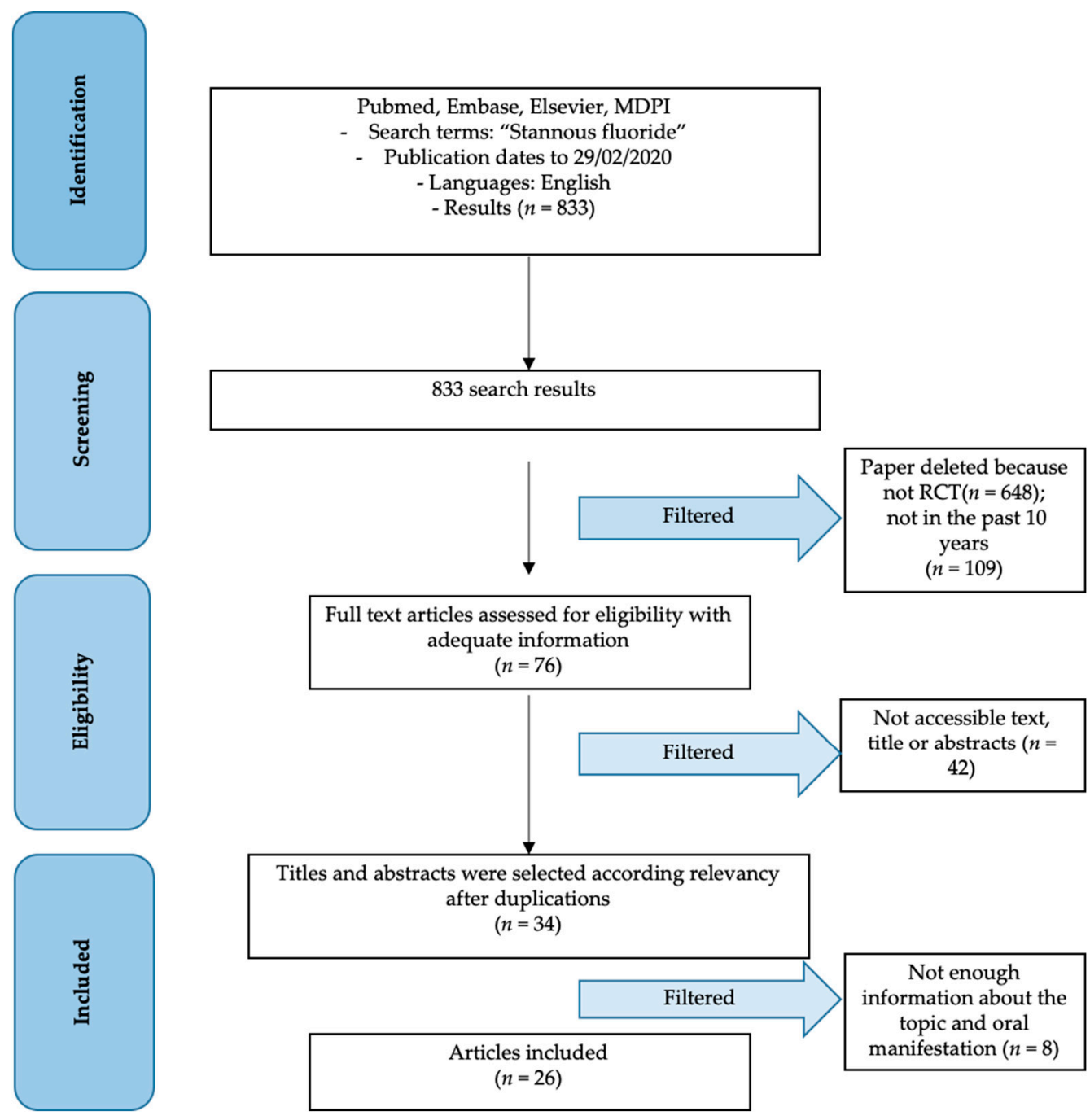

Figure 2. PRISMA flow chart.

\subsection{Study Characteristics} Table 1

Study characteristics have been shown according to material and methods section and showed in

\subsection{Risk of Bias within Studies}

Risk of bias analysis for each study has been showed in Table 2 according to Materials and Methods section guidelines.

\subsection{Results of Individual Studies}

West et al. [33] in their double blinded RCT, demonstrated how the use of stannous fluoride could significantly reduce enamel loss compared to $0.24 \%$ sodium fluoride dentifrice and a $0.3 \%$ triclosan one. Participants wore an intraoral appliance with two polished human enamel samples for six hours a day. They used assigned dentifrice two times a day and swished orange juice for 10 min a day. 
Seriwatanachai et al. [34] in a double blinded RCT evaluated the use of three different toothpastes. Stabilized $\mathrm{SnF}_{2}$ vs. $\mathrm{SnF}_{2}$ with zinc lactate vs. a fluoride dentifrice. They provided instruction and randomly assigned different dentifrices to patients. At three visits (0, 3 and 6 months) gingival and plaque index were evaluated. Both $\mathrm{SnF}_{2}$ toothpastes provided an index reduction in patients. Luo et al. [35] evaluated sensitivity reduction after an in-office bleaching treatment with different dentifrices after the use of stannous fluoride dentifrices or placebo ones. Tooth sensitivity was evaluated by a Visual Analog Scale (VAS) at day zero, day one, 2, 7, 14 and day 30. Immediately after bleaching the mean VAS values were lower in group 1, with no differences between 2 and 3 . Furthermore at days two, seven, 14 and 30 there were no statistical differences. Li et al. [36] in their in vitro blinded study evaluated the use of three different paste/gels on tooth stain reduction. $\mathrm{SnF}_{2}$ with $1 \%$ zinc paste showed the best results with a $17.5 \%$ better reduction at three weeks and a $27.8 \%$ reduction at the six-week time. According to Li et al. [36] stabilized Stannous Fluoride toothpaste performs better than non-abrasive toothpaste in this in vitro study; this toothpaste offers a therapeutic dentifrice with good performance in stain prevention and removal. In this case the staining agent consisted of a coffee, wine and tea (1:1:1) combination, used in hot water for $10 \mathrm{~min}$. Ionta et al. [37] in their in vitro study, evaluated the use of three different toothpastes with a fourth placebo group. The calcium silicate, sodium phosphate, and $1450 \mathrm{ppm}$ sodium monofluorophosphate showed better result in enamel wear, after an erosive and abrasive treatment. This study has been conducted on bovine enamel blocks. The investigated dentifrice reduced enamel loss against the acid challenge but had no effect against the acid and brushing challenge. In another double-blinded study of $\mathrm{Hu}$ et al. [38], it could be obtained how $\mathrm{SnF}_{2}$ toothpaste provides better results than common fluoride dentifrices. $\mathrm{SnF}_{2}$ toothpaste helps to reduce the inflammation index and to gain better plaque control. Haraszthy et al. [39] evaluated the effect of fluoride toothpaste on bacteria reduction, with promising results. Hagenflield et al. [40] in their study, evaluated differences in the microbiome after the use of two anti-adhesive and antibacterial toothpastes during periodontal therapy on a mild and moderate periodontitis population. They did not notice any differences in microbiome diversity. Creeth et al. [41] investigated in a short-term clinical study, how a stannous fluoride toothpaste could reduce DH compared to brushing with a conventional toothpaste after single use. This toothpaste, effectively, reduced DH on evaporative and tactile stimuli after a three-day treatment. It provided a significant Schiff sensitivity score and a tactile score reduction. Zero et al. [42] showed that all 1400-1450 ppm F dentifrices provide better remineralization than placebo, except for the $\mathrm{SnF}_{2}$ group. According to West et al. [43] there is a high grade of tubule occlusion in the toothpaste containing $0.454 \%$ stannous fluoride and in the fluoride toothpaste containing $0.76 \%$ sodium monofluorophosphate brushed dentine samples. There are no statistical differences between the first and second group of study. According to Frese et al. [44] an increase of caries-free surface between groups was observed. They highlighted a decrease of caries superficialis and media too. This four-year RCT was conducted on a population of athletes. According to authors, it could be shown how the odds of developing caries media on a new surface was significantly lower. In their RCT, West et al. [45] made 33 subjects wear an appliance with human enamel. They subdivided the sample into two groups, a $\mathrm{SnF}_{2}$ group and a triclosan group. The group 1 provided statistically significant better results about enamel loss from day 10 . A previous study of West et al. [46] provided valuable in vitro and in situ results about $\mathrm{SnF}_{2}$ toothpaste. Their human enamel samples showed a greater protection against dental erosion if $\mathrm{SnF}_{2}$ toothpaste was used instead of a sodium monofluorophosphate (SMFP)/arginine dentifrice. Marchetti et al. [47] evaluated plaque regrowth on a three-day mouthwash program. The differences between the groups were statistically significant, in particular, the use of an alcohol-free essential oil provided the worst results on plaque formation. The best result was provided by the use of a CHX mouthwash twice daily, followed by $\mathrm{SnF}_{2}$ with zinc mouthwashes. Geidel et al. [48] obtained significant results about API and OHI reduction in all groups. However, herbal toothpaste resulted in significantly lower API and OHI. Hove et al. [49] in their study, evaluated the effect of four different toothpastes on human molars, mounted on mouth appliances and worn by eight volunteers for nine days. To mimic the gastric effect, the specimens 
were etched for two minutes a day (extra-orally). The $\mathrm{SnF}_{2}$ specimens showed significantly lower enamel wear than the control group (fluoride free toothpaste, group 1) and group 4 with the same toothpaste of control and an additional $0.4 \% \mathrm{SnF}_{2}$ solution for two minutes. This solution fully protected enamel surface, better than the $0.4 \% \mathrm{SnF}_{2}$ toothpaste (group 2) and $0.454 \% \mathrm{SnF}_{2}$ toothpaste (group 3). Bellamy et al. [50] demonstrated how a $\mathrm{SnF}_{2}$ toothpaste can protect from enamel loss and that there is no differences between group 1 and 3. Another Bellamy et al. study [51] evaluated the plaque inhibition effect of $\mathrm{SnF}_{2} / \mathrm{NaF}$ dentifrice, they demonstrated how in a 17-day period this dentifrice could provide significant results on plaque inhibition. Stenhagen et al. [52] evaluated enamel resistance to erosive/abrasive with the use of different agents. Sodium fluoride, stannous fluoride, titanium tetrafluoride was compared to a control group mouth rinse. Human enamel specimens with one amalgam filling were used, and worn for nine days by volunteers. According to authors $\mathrm{SnF}_{2}$ and $\mathrm{TiF}(4)$ had better results. According to Jentsch et al. [53] there are significant differences between different oral rinses against plaque regrowth. They compared the use of essential oils, amine/stannous fluoride and chlorhexidine digluconate. CHX had better results on plaque thickness and cocci and bacilli counts already after $24 \mathrm{~h}$. West et al. [54], in their randomized crossover trials, compared stannous fluoride and essential oil mouth rinse. They used two different experimental stannous fluoride compositions and evaluated tooth and tongue staining. These formulations obtained good results on teeth staining vs. essential oils or water. Fine et al. [55] evaluated the effect of three dentifrices formulations. They collected samples from four sites, plaque, saliva, tongue and buccal mucosa, and evaluated six microbial types, anaerobes, Streptococci, Actinomyces, hydrogen-sulphide $\left(\mathrm{H}_{2} \mathrm{~S}\right)$-producing bacteria, Fusobacteria and Veillonella. The use of sodium fluoride/triclosan/copolymer (TCN/C) demonstrated reduction on microbiota. $\mathrm{SnF}_{2} / \mathrm{SHMP}$ showed significant reduction compared with the NaF group. Huysmans et al. [56] demonstrated on worn human enamel samples, how $\mathrm{SnF}_{2}$ toothpastes could reduce enamel erosion. Authors conducted a scanning electron microscope (SEM) analysis on enamel samples. Group 1 reduced erosive wear by $34 \%$ and group 2 by $26 \%$ with a significant difference to the control group (group 3). Wigger-Alberti et al. [57] evaluated the efficacy of different mouthrinses formulation in reducing oral malodor. They compared $250 \mathrm{ppm} \mathrm{F(-)amine} \mathrm{fluoride/stannous} \mathrm{fluoride}$ (ASF), $0.2 \%$ zinc lactate, oral malodour counteractives versus chlorhexidine mouthrinses (group two $0.05 \%$ CHX, $0.05 \%$ cetylpyridinium chloride, $0.14 \%$ zinc lactate; group three: mouthrinse III $(0.12 \%$ CHX) and with a control group (tap water). ASF mouthrinse showed a better effect on discoloration and organoleptic ratings. All data about groups of the cited studies in this paragraph have been shown in Table 1.

\subsection{Additional Analysis}

Through the use of a Mann-Whitney $U$ Test, it is possible to evaluate statistical differences between obtained data. Unfortunately, these data are inhomogeneous, and only an enamel wear loss statistical analysis has been performed (Table 4).

On eight studies, one study has been removed due to bovine enamel blocks use and another one for incomplete data. It is possible to demonstrate that the Critical value of $U$ at $p<0.05$ is 11 . Therefore, the result is significant at $p<0.05$. The $p$-value is 0.04648 . There is a significant difference between $\mathrm{SnF}_{2}$ use (toothpaste or mouthwashes) versus control groups on enamel wear loss. The result is significant at $p<0.05$.

\subsection{Risk of Bias Across Studies}

The studies taken into consideration in this review, being current, are few. The results obtained from this review may be skewed by an incorrect assessment within the studies and across the studies. The results were analyzed according to the methods listed in the previous chapter. Unfortunately, it was not possible to carry out a statistical analysis on the few data present and compare them to each other due to a lack of homogeneity of the measurements. However, none of the studies analyzed 
showed missing data or selective reporting. Surely it would have been an advantage to have a double blind when studying the samples.

It is also necessary to report that some studies could be influenced by an unreported conflict of interest, also given the important commercial nature of the study. The evaluated studies have investigated animal models, succeeding in demonstrating $\mathrm{SnF}_{2}$ products effects. The risk of bias is defined as low. It is preferable to use simple approaches for assessing validity that can be fully reported.

\section{Discussion}

\subsection{Summary of Evidence}

The literature articles provide useful information to clarify the functions and effects of this chemical compound in toothpastes. A discussion of the results, reporting here a critique of the conclusions of the individual scientific articles has been carried out. The use of different compounds in toothpastes has been much debated, mainly due to the presence of chemicals that could have more or less serious health effects. For example, orange juice and other citrus juices are perceived with an unpleasant taste after using toothpaste since the chemical interaction between the stannous fluoride present in the toothpaste and the acetic acid contained in the juice causes an alteration of the flavor. Sodium lauryl sulphate alters the taste perception, generally increasing the bitter taste, breaking down the phospholipids that inhibit the receptors of this taste; it is thought to also inhibit sweet taste receptors. On the other hand, the apple is known to have a pleasant flavor after the use of toothpaste. It is still an unsolved problem to distinguish whether the cause of the alteration of the orange juice taste is due to stannous fluoride or sodium lauryl sulfate; it is thought that the added aroma (often menthol) could also take part in the alteration of the perception of taste, when it is binding in the lingual receptors of the cold [58]. Fluoride is used in most toothpastes as an active ingredient. Many controversies arose as a result of the daily use of this substance. Fluoride toothpaste seems to cause damage to health: in fact, the intake of quantities of fluorine greater than $2 \mathrm{mg}$ per day causes fluorosis. If a large amount of toothpaste is ingested, a poison control center should be contacted immediately. In the USA, a wording is required on the tubes of toothpaste, inviting to contact a doctor or a control center, in case of ingestion of an excessive amount of toothpaste, and therefore of fluorine [59-61]. The effectiveness of fluorine is questioned by some chemists and scholars from all over the world, who appeal above all to an increasing number of studies that would highlight the toxicity of fluorine salts, capable of causing, with minimal overdoses, of fluorosis; ruining bones and teeth, and causing nervous system problems and cognitive deficits [62]. However, the articles cited refer to the toxicity of fluorine food supplements, without prejudice to the usefulness of the fluorine contained in toothpastes which instead performs an effective protective activity. In this context, it is worth underlining that the availability of efficient antiseptic systems, without particular contraindications, can be indicated for pregnant women, as it limits the phenomenon of premature births and therefore of underweight babies that recent studies hypothesize to be related to the presence of gingivitis, pathologies that causes the release into the circulation of factors, such as metalloproteases, capable of interacting with the hormonal systems and consequently stimulating early uterine contractions. There are no allergic reactions reported in results. Other scholars counter these criticisms, stating instead that the advantages of using fluorine as an aid in the mineralization of dental enamel are also demonstrated by natural observations in populations residing in neighboring areas in the presence of waters rich in fluorine ions, in which it is present a low incidence of caries. It is also noteworthy that the risk of using fluoride is quite low and indeed the use of toothpaste with a high fluoride content (1350-1500 ppm) is recommended for all ages (although smaller volumes are used for children small; a "stain" of toothpaste up to 3 years) [63-65]. There are several fluoride-free toothpaste options available on the market for those who choose not to use this element. There are commercially available toothpastes based on delicate washing substances (sodium cocoglucoside tartrate, white clay) and soothing plant extracts for gums and periodontal health (extract of myrrh, chamomile, krameria triandra), peppermint oil, strawberry plant extract. 
Some natural toothpastes use carboxymethylchitosan, present in the exoskeleton of crustaceans, as an anticaries active ingredient [66]. This homoglycan is able to bring the $\mathrm{pH}$ values of the mouth closer to neutrality by neutralizing lactic acid; it also has antimicrobial properties. Stannous fluoride showed good antibacterial properties in animal studies too. It is worth mentioning, even if briefly, some results that were excluded from the research phase because they did not meet the criteria (older than 10 years). A study by Yu et al. [67] evaluated the potential to reduce enamel and dentin erosion with a single application of stannous chloride-containing fluoride solution. This was assessed on both enamels covered by the enamel film and dentin under acidic conditions in vitro. According to the authors the staus chloride-containing fluoride solution reduced calcium loss of enamel and dentine to up to 6 and $3.5 \mathrm{~min}$, respectively. Even a single application therefore showed positive effects. Wang et al. [68] investigated the fluoride-releasing ability of a tooth separator consisting of elastomer and fluoride. The enamel area contacting with the separator and its surrounding area showed lower mineral loss compared to control with no tooth separator application. Stookey et al. [69] evaluated the anticaries effectiveness of a low-dose sodium fluoride dentifrice in 2004. In this clinical trial authors indicated that while no difference in caries increments was observed between the low-NaF and control groups with high-NaF and the $\mathrm{SnF}_{2}-\mathrm{HMP}$ and control groups. Orbak et al. [70] examined the effect of an electro-ionizing toothbrush with stannous fluoride in the treatment of dentin hypersensitivity. Authors showed how an ionizing brush may be an effective tool for treatment of dentin hypersensitivity in the post-periodontal surgery.

According to Seltzer et al. [71] stannous fluoride products (BacDerm; Emerald 3 Enterprises Inc., Camdenton, MO, USA) can significantly reduce a bacterial skin infection. This product showed significant reduction on hair coat, odour, pruritus on dogs. Toothpastes containing amine fluoride and stannous fluoride perform remineralizing action by forming a precipitate of calcium fluoride, adhering to the tooth surface, capable of subsequently releasing fluorine particles (Figure 1). Stannous fluoride has excellent antiplaque and antibacterial potential. In fact, it is able to penetrate the bacterial membrane, accumulating inside the cell and inhibiting the activity of the bacterial enzymes involved in the production of acids from sugar. Thanks to its natural acidity, in aqueous solutions it causes a reduction in the $\mathrm{pH}$ inside the bacterial cells, also preventing the activity of other enzymes [72-76].

In addition, it appears that stannous fluoride is able to block interactions between the bacteria themselves and between the bacteria and the surface of the teeth. In this way it prevents bacteria from accumulating on the tooth surfaces. However, the use of stannous fluoride as an active ingredient in oral hygiene products presents some difficulties. The divalent ions of which it is composed are very reactive: in aqueous environments and in the presence of oxygen they dissociate within a few hours to form a white precipitate; the ions oxidize to form tetravalent tin ions $\left(\mathrm{Sn}_{4}+\right)$. Unfortunately, these reactions, which occur in the presence of water and oxygen, also lead to the inactivation of stannous fluoride, reducing its antibacterial effects. The imperfections on the enamel surface are filled with micrometric aggregates of apatites nanocrystals forming a biomimetic protective coating. This also repairs dentin and reduces dentinal sensitivity.

\subsubsection{Hard Tissue Effects of Stannous Fluoride Compounds}

According to West et al. [33] stannous fluoride dentifrice shows a greater erosion protection relative to the $\mathrm{NaF} /$ triclosan compositions. Ionta et al. [37], in their conducted study on bovine enamel block, showed how calcium silicate, sodium phosphate, and 1450 ppm sodium monofluorophosphate dentifrice could reduce enamel loss against the acid challenge. $\mathrm{Hu}$ et al. demonstrated how a $\mathrm{SnF} 2$ dentifrice improves clinical outcomes and how in the six month-period time evaluation, it provided an improvement in all evaluated indexes. West et al. [45] demonstrated how a stabilized $\mathrm{SnF}_{2}$ dentifrice provides superior protection against tooth enamel surface loss compared to $\mathrm{NaF} /$ triclosan dentifrice. The triclosan/copolymer technology is compatible with the fluoride to which it can be associated in toothpaste formulations. In another study, West et al. [46] confirmed the superiority of stabilized $\mathrm{SnF}_{2}$ dentifrice for protecting human teeth from erosion. According to Hove et al. [49] 0.4\% $\mathrm{SnF}_{2}$ solution 
mouthwash, after a fluoride-free toothpaste brushing, provided better results on enamel protection, better than $\mathrm{SnF}_{2}$ toothpastes too. $\mathrm{SnF}_{2}$ toothpaste could protect enamel from erosion according to Bellamy et al. [50]. $\mathrm{SnF}_{2}$ toothpaste provided better results than the $\mathrm{NaF}$ dentifrice or control group (water). Stenhagen et al. [52] demonstrated how $\mathrm{SnF}_{2}$ mouth rinse could reduce enamel erosion if compared to $\mathrm{TiF}_{4}$ and $\mathrm{NaF}$ in human enamel samples. Huysmans et al. [56] with their SEM analysis concluded that $\mathrm{SnF}_{2}$ toothpaste could reduce erosive tooth wear in situ. West et al. [43] concluded that there are no differences in tubule occlusion capability between toothpaste containing $0.454 \%$ stannous fluoride and fluoride toothpaste containing $0.76 \%$ sodium monofluorophosphate. However, following the acid challenge, there was a statistically significantly greater degree of occlusion in the stannous fluoride toothpaste.

\subsubsection{Biological and Plaque Effects of Stannous Fluoride Compounds}

Haraszthy et al. [39] concluded that stannous fluoride dentifrice provides a bacteria reduction $12 \mathrm{~h}$ after brushing, and microbial reduction continues four hours later, with promising result on long term use. According to Hagenfield et al. [40] the use of a toothpaste with anti-adhesive zinc-substituted carbonated hydroxyapatite did not provide changes on microbial composition versus anti-adhesive amine fluoride/stannous fluoride, but authors evaluated microbial composition after oral hygiene and periodontal therapy. Marchetti et al. [47] in their randomized crossover clinical trial, proved how, CHX mouthwashes provide better results against plaque regrowth compared to alcohol-free essential oil or $\mathrm{SnF}_{2}$ mouthwashes. Geidel et al. [48] in their study, concluded that after 24 weeks of controlled study, the herbal toothpaste, compared to a stannous fluoride toothpaste, was as good as the control toothpaste, with no side effect. In terms of periodontal health, the herbal toothpaste could be a suitable alternative to conventional dentifrices. Bellamy et al.'s [51] population showed less plaque coverage with the use of $\mathrm{SnF}_{2}$ toothpaste at a 17-day period than a fluoride dentifrice. Jentsch et al. [53] demonstrated how chlorhexidine digluconate mouth rinses could reduce plaque thickness and counts of cocci and bacilli after use, it obtained better results if compared to essential oils and amine/stannous fluoride. These last two groups did not differ between us. Fine et al. [55] demonstrated that TCN/C dentifrice formulation consistently reduced for a range of microorganisms in diverse oral sites in comparison with the $\mathrm{NaF}$, or the $\mathrm{SnF}_{2} / \mathrm{SHMP}$ dentifrice formulations as seen $12 \mathrm{~h}$ after brushing. Seriwatanachai et al. [34] showed how $\mathrm{SnF}_{2}$ dentifrice could help patients against plaque formation and a gingival index reduction.

\subsubsection{Oral Health Related Quality of Life Effects of Stannous Fluoride Compounds}

According to Li et al. [36] stabilized stannous fluoride toothpaste performs better on tooth staining removal than regular fluoride toothpastes. Lorenz et al. [49] performed a RCT, in healthy dental students, with no mechanical oral hygiene and eight daily rinses with mouth rinse and black tea. They evaluated the effect of three different rinses on five groups: $\mathrm{AmF} / \mathrm{SnF}_{2}$ rinse (3 groups), essential oil rinse and water. All rinses led to tooth and tongue staining, with statistical differences in tooth staining between groups. According to obtained results, group number 3 had promising potential on less tooth discoloration than other $\mathrm{AmF} / \mathrm{SnF}_{2}$ rinses. Lorenz et al. [49] proved that one of the experimental $\mathrm{AmF} / \mathrm{SnF}_{2}$ rinses leads to less staining than others. West et al. [54] concluded that stannous fluoride mouth rinses could provide good results on teeth staining formation. According to Wigger-Alberti et al. [57], ASF products showed better results than CHX products on discoloration and oral malodour (organoleptic score and volatile sulfur compounds). Luo et al. [35], comparing stannous fluoride group with potassium nitrate and placebo, affirmed that the use of potassium nitrate could alleviate tooth sensitivity during and after in-office bleaching. Creeth et al. [41] demonstrated how brushing with an experimental anhydrous $0.454 \% \mathrm{SnF}_{2}$ polyphosphate toothpaste reduced $\mathrm{DH}$ with a single use and better in three days use. 


\section{Limitations}

The main limitation of this study is given by the fact that it is not possible to couple the single results and carry out a univocal statistical analysis, as the results come in the single results evaluated using different parameters. Data from the last ten years were considered in order not to create discrepancies between types of experimentation and between different chemical formulations of toothpaste. Only English-language articles were considered and this may be a limitation.

\section{Conclusions}

Although it was not possible to perform a meta-analysis due to the incomparable results, it is evident how much the individual studies support the use of compounds with stannous fluoride for oral hygiene. This compound has demonstrated different functions and characteristics, in the absence of reported contraindications. The contraindications in fact, are those for fluorine, from overdose. The stannous fluoride has been shown to have positive effects both in terms of plaque formation, tooth stains and gingival inflammation. The antimicrobial effects of staus fluoride are often overcome by other components such as chlorhexidine. As for the other outcomes, the results appear to be promising versus sodium fluoride, herbal toothpaste or triclosan products. A meta-analysis on enamel loss has been conducted, it shows that $\mathrm{SnF}_{2}$ provides better results with $p<0.05$ value on enamel wear loss than in control groups. Furthermore, it appears to have excellent results with respect to the remineralization of the enamel, even in the presence of demineralized areas, or in the occlusion of the exposed dentinal tubules.

Author Contributions: Conceptualization, M.C. and L.F.; methodology, L.F.; software, L.F.; validation, G.C.; formal analysis, L.F.; investigation, G.C.; data curation, L.F.; writing—original draft preparation, L.F.; writing-review and editing, G.C.; visualization, A.S.H. and L.L.; supervision, M.C.; project administration, M.C. All authors have read and agreed to the published version of the manuscript.

Funding: This research received no external funding.

Conflicts of Interest: The authors declare no conflict of interest.

\section{References}

1. Diesendorf, M. The mystery of declining tooth decay. Nature 1986, 322, 125-129. [CrossRef] [PubMed]

2. Anderson, C. Fluoride given the all clear. Nature 1991, 349, 732. [CrossRef] [PubMed]

3. Jones, D. Dentistry's Leading Edge. Available online: https://web.archive.org/web/20100826062103/http: //www.drdavidjones.com/qa.htm (accessed on 9 August 2020).

4. Horowitz, H.S. Proper use of fluoride products in fluoridated communities. Lancet 1999, 353, 1462. [CrossRef]

5. Hargreaves, J.A. Fluoride toothpaste. Lancet 1971, 2, 929. [CrossRef]

6. Dahl, A.R.; Hodgson, E. Complexes of stannous fluoride and other group IVB dihalides with mammalian hemoproteins. Science 1977, 197, 1376-1378. [CrossRef]

7. Dental Association: Toothpaste "Recognition" Subject of Controversy. Science 1961, 134, 1349. [CrossRef]

8. Šket, T.; Kukec, A.; Kosem, R.; Artnik, B. The history of public health use of fluorides in caries prevention. Zdr Varst 2017, 56, 140-146. [CrossRef]

9. Horowitz, H.S. Fluoride and enamel defects. Adv. Dent. Res. 1989, 3, 143-146. [CrossRef]

10. Cury, J.A.; Ricomini-Filho, A.P.; Berti, F.L.P.; Tabchoury, C.P. Systemic Effects (Risks) of Water Fluoridation. Braz. Dent. J. 2019, 30, 421-428. [CrossRef]

11. Arora, S.; Kumar, J.V.; Moss, M.E. Does water fluoridation affect the prevalence of enamel fluorosis differently among racial and ethnic groups? J. Public Health Dent. 2018, 78, 95-99. [CrossRef]

12. Oliveira, M.R.C.; Oliveira, P.H.C.; Oliveira, L.H.C.; Horliana, A.; César, P.F.; Moura, S.K.; Bussadori, S.K. Microhardness of bovine enamel after different fluoride application protocols. Dent. Mater. J. 2019, 38, 61-67. [CrossRef] [PubMed]

13. Magalhães, A.C.; Wiegand, A.; Rios, D.; Buzalaf, M.A.R.; Lussi, A. Fluoride in dental erosion. Monogr. Oral Sci. 2011, 22, 158-170. [CrossRef] [PubMed] 
14. Limeback, H. Enamel formation and the effects of fluoride. Community Dent. Oral Epidemiol. 1994, 22, 144-147. [CrossRef] [PubMed]

15. Kalsbeek, H. Series: Caries prevention in historical perspective. Fluoride. Ned. Tijdschr. Tandheelkd. 2018, 125, 257-261. [CrossRef]

16. Koenigs, P.M.; Faller, R.V. Fluoride's Mechanism of Action. Available online: https://www.dentalcare.com/enus/professional-education/ce-courses/ce410/fluoride-s-mechanism-of-action (accessed on 11 February 2020).

17. Riva, M.A. Advertising: Giacomo Puccini in dental history. Br. Dent. J. 2018, 225, 684. [CrossRef]

18. Matthews, D.C. Prevention and treatment of periodontal diseases in primary care. Evid. Based Dent. 2014, 15, 68-69. [CrossRef]

19. Katanec, T.; Majstorovic, M.; Negovetic Vranic, D.; Ivic Kardum, M.; Marks, L.A. New toothpaste to deal with dentine hypersensitivity: Double-blind randomized controlled clinical trial. Int. J. Dent. Hyg. 2018, 16, 78-84. [CrossRef]

20. Stookey, G.K.; Stahlman, D.B. Enhanced fluoride uptake in enamel with a fluoride-impregnated prophylactic cup. J. Dent. Res. 1976, 55, 333-341. [CrossRef]

21. Richards, A.; Fejerskov, O.; Baelum, V. Enamel fluoride in relation to severity of human dental fluorosis. Adv. Dent. Res. 1989, 3, 147-153. [CrossRef]

22. Pendrys, D.G.; Stamm, J.W. Relationship of total fluoride intake to beneficial effects and enamel fluorosis. J. Dent. Res. 1990, 69, 5295-5338. [CrossRef]

23. Pai, N.; McIntyre, J.; Tadic, N.; Laparidis, C. Comparative uptake of fluoride ion into enamel from various topical fluorides in vitro. Aust. Dent. J. 2007, 52, 41-46. [CrossRef] [PubMed]

24. Muntean, A.; Sava, S.; Delean, A.G.; Mihailescu, A.M.; Dumitrescu, L.S.; Moldovan, M.; Festila, D.G. Toothpaste composition effect on enamel chromatic and morphological characteristics: In vitro analysis. Materials 2019, 12, 2610. [CrossRef]

25. Scribante, A.; Dermenaki Farahani, M.R.; Marino, G.; Matera, C.; Rodriguez y Baena, R.; Lanteri, V.; Butera, A. Biomimetic Effect of nano-hydroxyapatite in demineralized enamel before orthodontic bonding of brackets and attachments: Visual, adhesion strength, and hardness in in vitro tests. BioMed Res. Int. 2020, 2020, 6747498. [CrossRef]

26. Kim, H.-N.; Kong, W.-S.; Lee, J.-H.; Kim, J.-B. Reduction of dental caries among children and adolescents from a 15-year community water fluoridation program in a township area, Korea. Int. J. Environ. Res. Public Health 2019, 16, 1306. [CrossRef] [PubMed]

27. Cervino, G.; Fiorillo, L.; Herford, A.S.; Laino, L.; Troiano, G.; Amoroso, G.; Crimi, S.; Matarese, M.; D’Amico, C.; Nastro Siniscalchi, E.; et al. Alginate Materials and Dental Impression Technique: A Current State of the Art and Application to Dental Practice. Mar. Drugs 2018, 17, 18. [CrossRef] [PubMed]

28. EzEldeen, M.; Gizani, S.; Declerck, D. Long-term outcome of oral health in patients with early childhood caries treated under general anaesthesia. Eur. Arch. Paediatr. Dent. 2015, 16, 333-340. [CrossRef]

29. Cortelli, S.C.; Costa, F.O.; Rode Sde, M.; Haas, A.N.; Andrade, A.K.; Pannuti, C.M.; Escobar, E.C.; Almeida, E.R.; Cortelli, J.R.; Pedrazzi, V. Mouthrinse recommendation for prosthodontic patients. Braz. Oral Res. 2014, 28, 1-9. [CrossRef]

30. Savovic, J.; Turner, R.M.; Mawdsley, D.; Jones, H.E.; Beynon, R.; Higgins, J.P.T.; Sterne, J.A.C. Association between risk-of-bias assessments and results of randomized trials in cochrane reviews: The ROBES meta-epidemiologic study. Am. J. Epidemiol. 2018, 187, 1113-1122. [CrossRef]

31. Mansournia, M.A.; Higgins, J.P.; Sterne, J.A.; Hernan, M.A. Biases in randomized trials: A conversation between trialists and epidemiologists. Epidemiology 2017, 28, 54-59. [CrossRef]

32. Higgins, J.P.; Altman, D.G.; Gotzsche, P.C.; Juni, P.; Moher, D.; Oxman, A.D.; Savovic, J.; Schulz, K.F.; Weeks, L.; Sterne, J.A. The Cochrane Collaboration's tool for assessing risk of bias in randomised trials. BMJ 2011, 343, d5928. [CrossRef]

33. West, N.X.; He, T.; Hellin, N.; Claydon, N.; Seong, J.; Macdonald, E.; Farrell, S.; Eusebio, R.; Wilberg, A. Randomized in situ clinical trial evaluating erosion protection efficacy of a $0.454 \%$ stannous fluoride dentifrice. Int. J. Dent. Hyg. 2019, 17, 261-267. [CrossRef] [PubMed]

34. Seriwatanachai, D.; Triratana, T.; Kraivaphan, P.; Amaornchat, C.; Mateo, L.R.; Sabharwal, A.; Delgado, E.; Szewczyk, G.; Ryan, M.; Zhang, Y.P. Effect of stannous fluoride and zinc phosphate dentifrice on dental plaque and gingivitis: A randomized clinical trial with 6-month follow-up. J. Am. Dent. Assoc. 2019, 150, S25-S31. [CrossRef] [PubMed] 
35. Luo, Z.Q.; Zhang, Y.; Tang, L.; Liu, Y.H. Clinical evaluation of the effect of reducing tooth sensitivity caused by in office bleaching using dentifrices. Beijing Da Xue Xue Bao Yi Xue Ban 2019, 51, 340-344.

36. Li, Y.; Suprono, M.; Mateo, L.R.; Zhang, Y.P.; Denis, J.; D’Ambrogio, R.; Sullivan, R.; Thomson, P. Solving the problem with stannous fluoride: Extrinsic stain. J. Am. Dent. Assoc. 2019, 150, S38-S46. [CrossRef] [PubMed]

37. Ionta, F.Q.; Dos Santos, N.M.; Mesquita, I.M.; Dionísio, E.J.; Cruvinel, T.; Honório, H.M.; Rios, D. Is the dentifrice containing calcium silicate, sodium phosphate, and fluoride able to protect enamel against chemical mechanical wear? An in situ/ex vivo study. Clin. Oral Investig. 2019, 23, 3713-3720. [CrossRef] [PubMed]

38. Hu, D.; Li, X.; Liu, H.; Mateo, L.R.; Sabharwal, A.; Xu, G.; Szewczyk, G.; Ryan, M.; Zhang, Y.P. Evaluation of a stabilized stannous fluoride dentifrice on dental plaque and gingivitis in a randomized controlled trial with 6-month follow-up. J. Am. Dent. Assoc. 2019, 150, S32-S37. [CrossRef]

39. Haraszthy, V.I.; Raylae, C.C.; Sreenivasan, P.K. Antimicrobial effects of a stannous fluoride toothpaste in distinct oral microenvironments. J. Am. Dent. Assoc. 2019, 150, S14-S24. [CrossRef]

40. Hagenfeld, D.; Prior, K.; Harks, I.; Jockel-Schneider, Y.; May, T.W.; Harmsen, D.; Schlagenhauf, U.; Ehmke, B. No differences in microbiome changes between anti-adhesive and antibacterial ingredients in toothpastes during periodontal therapy. J. Periodontal Res. 2019, 54, 435-443. [CrossRef]

41. Creeth, J.; Gallob, J.; Sufi, F.; Qaqish, J.; Gomez-Pereira, P.; Budhawant, C.; Goyal, C. Randomised clinical studies investigating immediate and short-term efficacy of an occluding toothpaste in providing dentine hypersensitivity relief. BMC Oral Health 2019, 19, 98. [CrossRef]

42. Zero, D.T.; Lippert, F.; Hara, A.T.; Creeth, J.E.; Newby, E.E.; Butler, A.; Constantin, P.; Bosma, M.L. In situ anticaries efficacy of dentifrices with different formulations-A pooled analysis of results from three randomized clinical trials. J. Dent. 2018, 77, 93-105. [CrossRef]

43. West, N.X.; Seong, J.; Hellin, N.; Macdonald, E.L.; Jones, S.B.; Creeth, J.E. Assessment of tubule occlusion properties of an experimental stannous fluoride toothpaste: A randomised clinical in situ study. J. Dent. 2018, 76, 125-131. [CrossRef] [PubMed]

44. Frese, C.; Wohlrab, T.; Sheng, L.; Kieser, M.; Krisam, J.; Frese, F.; Wolff, D. Clinical management and prevention of dental caries in athletes: A four-year randomized controlled clinical trial. Sci. Rep. 2018, 8, 16991. [CrossRef] [PubMed]

45. West, N.X.; Seong, J.; Hellin, N.; Eynon, H.; Barker, M.L.; He, T. A clinical study to measure anti-erosion properties of a stabilized stannous fluoride dentifrice relative to a sodium fluoride/triclosan dentifrice. Int. J. Dent. Hyg. 2017, 15, 113-119. [CrossRef] [PubMed]

46. West, N.X.; He, T.; Macdonald, E.L.; Seong, J.; Hellin, N.; Barker, M.L.; Eversole, S.L. Erosion protection benefits of stabilized $\mathrm{SnF}(2)$ dentifrice versus an arginine-sodium monofluorophosphate dentifrice: Results from in vitro and in situ clinical studies. Clin. Oral Investig. 2017, 21, 533-540. [CrossRef]

47. Marchetti, E.; Casalena, F.; Capestro, A.; Tecco, S.; Mattei, A.; Marzo, G. Efficacy of two mouthwashes on 3-day supragingival plaque regrowth: A randomized crossover clinical trial. Int. J. Dent. Hyg. 2017, 15, 73-80. [CrossRef]

48. Geidel, A.; Krüger, M.; Schrödl, W.; Jentsch, H. Control of Plaque and Gingivitis by an Herbal Toothpaste A Randomised Controlled Study. Oral Health Prev. Dent. 2017, 15, 407-413. [CrossRef] [PubMed]

49. Lorenz, K.; Noack, B.; Herrmann, N.; Hoffmann, T. Tooth staining potential of experimental amine fluoride/stannous fluoride mouth rinse formulations-a randomized crossover forced staining study. Clin. Oral Investig. 2015, 19, 1039-1045. [CrossRef]

50. Hove, L.H.; Stenhagen, K.R.; Holme, B.; Tveit, A.B. The protective effect of $\mathrm{SnF}_{2}$ containing toothpastes and solution on enamel surfaces subjected to erosion and abrasion in situ. Eur. Arch. Paediatr. Dent. 2014, 15, 237-243. [CrossRef]

51. Bellamy, P.G.; Harris, R.; Date, R.F.; Mussett, A.J.; Manley, A.; Barker, M.L.; Hellin, N.; West, N.X. In situ clinical evaluation of a stabilised, stannous fluoride dentifrice. Int. Dent. J. 2014, 64 (Suppl. 1), 43-50. [CrossRef]

52. Bellamy, P.G.; Boulding, A.; Farmer, S.; Day, T.N.; Barker, M.L.; Harris, R.; Mussett, A.J. Randomized in vivo trial evaluating plaque inhibition benefits of an advanced stannous-containing sodium fluoride dentifrice used in conjunction with power brush technology. Int. J. Dent. Hyg. 2014, 12, 89-95. [CrossRef]

53. Stenhagen, K.R.; Hove, L.H.; Holme, B.; Tveit, A.B. The effect of daily fluoride mouth rinsing on enamel erosive/abrasive wear in situ. Caries Res. 2013, 47, 2-8. [CrossRef] [PubMed] 
54. Jentsch, H.; Mozaffari, E.; Jonas, L. Scanning electron microscopy of growing dental plaque: A quantitative study with different mouth rinses. Ultrastruct. Pathol. 2013, 37, 233-240. [CrossRef] [PubMed]

55. West, N.X.; Addy, M.; Newcombe, R.; Macdonald, E.; Chapman, A.; Davies, M.; Moran, J.; Claydon, N. A randomised crossover trial to compare the potential of stannous fluoride and essential oil mouth rinses to induce tooth and tongue staining. Clin. Oral Investig. 2012, 16, 821-826. [CrossRef]

56. Fine, D.H.; Sreenivasan, P.K.; McKiernan, M.; Tischio-Bereski, D.; Furgang, D. Whole mouth antimicrobial effects after oral hygiene: Comparison of three dentifrice formulations. J. Clin. Periodontol. 2012, 39, 1056-1064. [CrossRef] [PubMed]

57. Huysmans, M.C.; Jager, D.H.; Ruben, J.L.; Unk, D.E.; Klijn, C.P.; Vieira, A.M. Reduction of erosive wear in situ by stannous fluoride-containing toothpaste. Caries Res. 2011, 45, 518-523. [CrossRef] [PubMed]

58. Wigger-Alberti, W.; Gysen, K.; Axmann, E.M.; Wilhelm, K.P. Efficacy of a new mouthrinse formulation on the reduction of oral malodour in vivo. A randomized, double-blind, placebo-controlled, 3 week clinical study. J. Breath Res. 2010, 4, 017102. [CrossRef] [PubMed]

59. Anil, S. Plasma cell gingivitis among herbal toothpaste users: A report of three cases. J. Contemp. Dent. Pract. 2007, 8, 60-66. [CrossRef]

60. Seymour, D.G.; Day, J.J.; Pathy, M.S. Sodium fluoride: Too late and too toxic for the elderly osteoporotic patient? Additional comments. Age Ageing 1990, 19, 425-426. [CrossRef]

61. Zohoori, F.V.; Innerd, A.; Azevedo, L.B.; Whitford, G.M.; Maguire, A. Effect of exercise on fluoride metabolism in adult humans: A pilot study. Sci. Rep. 2015, 5, 16905. [CrossRef]

62. Durbakula, K.; Prabhu, V.; Jose, M. Genotoxicity of non-alcoholic mouth rinses: A micronucleus and nuclear abnormalities study with fluorescent microscopy. J. Investig. Clin. Dent. 2018, 9, e12309. [CrossRef]

63. Shepherd, N.A.; Levison, D.A.; Heatley, R.V.; Braegger, C.P.; Corrigan, C.J.; Macdonald, T.T.; Ghoda, M.K. Toothpaste and Crohn's disease. Lancet 1990, 336, 1382. [CrossRef]

64. Ley, C.; Pischel, L.; Parsonnet, J. Triclosan and triclocarban exposure and thyroid function during pregnancy-A randomized intervention. Reprod. Toxicol. 2017, 74, 143-149. [CrossRef]

65. Herlofson, B.B.; Barkvoll, P. Oral mucosal desquamation caused by two toothpaste detergents in an experimental model. Eur. J. Oral Sci. 1996, 104, 21-26. [CrossRef] [PubMed]

66. Cicciù, M.; Fiorillo, L.; Cervino, G. Chitosan Use in Dentistry: A Systematic Review of Recent Clinical Studies. Mar. Drugs 2019, 17, 417. [CrossRef] [PubMed]

67. Yu, H.; Wegehaupt, F.J.; Zaruba, M.; Becker, K.; Roos, M.; Attin, T.; Wiegand, A. Erosion-inhibiting potential of a stannous chloride-containing fluoride solution under acid flow conditions in vitro. Arch. Oral Biol. 2010, 55, 702-705. [CrossRef]

68. Wang, X.Y.; Miyazaki, K.; Itoh, Y.; Motokawa, W. Effect of experimental fluoride-releasing tooth separator on acid resistance of human enamel in vitro. Dent. Mater. J. 2001, 20, 275-285. [CrossRef]

69. Stookey, G.K.; Mau, M.S.; Isaacs, R.L.; Gonzalez-Gierbolini, C.; Bartizek, R.D.; Biesbrock, A.R. The relative anticaries effectiveness of three fluoride-containing dentifrices in Puerto Rico. Caries Res. 2004, 38, 542-550. [CrossRef]

70. Orbak, R.; Canakçi, V.; Tezel, A. Clinical evaluation of an electron-ionizing toothbrush with a tooth paste containing stannous fluoride in treatment of dentine hypersensitivity following periodontal surgery. Dent. Mater. J. 2001, 20, 164-171. [CrossRef]

71. Seltzer, J.D.; Flynn-Lurie, A.K.; Marsella, R.; Brennan, M.M. Investigation of the clinical efficacy of $0.2 \%$ topical stannous fluoride for the treatment of canine superficial pyoderma: A prospective, randomized, double-blinded, placebo-controlled trial. Vet. Dermatol. 2010, 21, 249-258. [CrossRef]

72. Fiorillo, L.; De Stefano, R.; Cervino, G.; Crimi, S.; Bianchi, A.; Campagna, P.; Herford, A.S.; Laino, L.; Cicciù, M. Oral and Psychological Alterations in Haemophiliac Patients. Biomedicines 2019, 7, 33. [CrossRef]

73. Cicciù, M.; Herford, A.S.; Cervino, G.; Troiano, G.; Lauritano, F.; Laino, L. Tissue fluorescence imaging (VELscope) for quick non-invasive diagnosis in oral pathology. J. Craniofac. Surg. 2017, 28, e112-e115. [CrossRef]

74. Nastro, E.; Musolino, C.; Allegra, A.; Oteri, G.; Cicciù, M.; Alonci, A.; Quartarone, E.; Alati, C.; De Ponte, F.S. Bisphosphonate-associated osteonecrosis of the jaw in patients with multiple myeloma and breast cancer. Acta Haematol. 2007, 117, 181-187. [CrossRef] 
75. Isola, G.; Matarese, M.; Ramaglia, L.; Cicciù, M.; Matarese, G. Evaluation of the efficacy of celecoxib and ibuprofen on postoperative pain, swelling, and mouth opening after surgical removal of impacted third molars: A randomized, controlled clinical trial. Int. J. Oral Maxillofac. Surg. 2019, 48, 1348-1354. [CrossRef]

76. Cicciù, M.; Risitano, G.; Lo Giudice, G.; Bramanti, E. Periodontal health and caries prevalence evaluation in patients affected by Parkinson's disease. Parkinson's Dis. 2012. [CrossRef]

(C) 2020 by the authors. Licensee MDPI, Basel, Switzerland. This article is an open access article distributed under the terms and conditions of the Creative Commons Attribution (CC BY) license (http://creativecommons.org/licenses/by/4.0/). 